\title{
Application of My Country's Scientific and Technological Achievements in the Design of Cultural and Creative Products
}

\author{
Yonggang Ren, Jiaxing Wang \\ North China University of Technology, Shijingshan District, Beijing, 100144, China
}

\begin{abstract}
In recent years, the continuous growth of economic strength has promoted people's pursuit of culture, and the demand for cultural and creative products has increased year by year. Cultural and creative products are different from ordinary products. Ordinary products pursue functions, beauty and economic benefits, while cultural and creative products endow the concept of product culture. Cultural and creative products can represent culture, ideals, beliefs, etc. At present, China has entered a new era. China has made major achievements in various fields. These achievements are areas that are difficult for ordinary people to get involved. However, these achievements are really closely related to people's lives. How to let people understand these deep-seated areas and how to link ordinary people with high-tech achievements are the issues that this article focuses on. This article first sorts out the propaganda paths of various scientific and technological achievements nowadays, then analyzes their existing problems, proposes a design strategy for cultural and creative products based on scientific and technological achievements, and finally completes the design of cultural and creative products: These scientific and technological achievements can be designed into simple and easy-to-understand patterns, and then applied to the design of cultural and creative products such as T-shirts, which will provide impetus to telling Chinese stories, enhancing cultural strength and national self-confidence.
\end{abstract}

Keywords: Scientific and technological achievements, Cultural and creative product, Design, National self-confidence.

\section{Introduction}

In 2017, China entered a new era. Since then, China has made a series of great achievements and issued a series of great declarations, demonstrating to the world that China is a country with strong vitality. More than a hundred years ago, China was still very backward and was facing a situation of being carved up; a hundred years later, China was already at the forefront of the world. In 2020, China has completed its thirteenth "Five-Year Plan." During this year, major breakthroughs were achieved in scientific explorations such as the "Chang'e 5" lunar probe program, the "Fighter" deepsea exploration, the "BeiDou Navigation Satellite System", and the "China Skyeye" Five-hundred-meter Aperture Spherical radio Telescope. These results are undoubtedly huge, but for ordinary people, it seems too far away. In fact, these results have been applied to ordinary people. To sort out the shortcomings in the publicity of scientific and technological achievements in recent years, Sorting out the shortcomings in the publicity of scientific and technological achievements in recent years, and linking the public with high-tech achievements by designing cultural and creative products can stimulate the enthusiasm and yearning of the whole society, especially the young people, for science and technology, and enhance their national self-confidence.

\section{The Publicity Path of Scientific and Technological Achievements and Their Shortcomings}

The publicity path of scientific and technological achievements can be divided into two forms: online and offline: There are offline scientific and technological achievements, science and cultural activities and scientific and technological achievements patriotism education base; Online activities are mainly based on popular science short videos.

\subsection{Popularization Activities of Scientific and} Technological Achievements

The popularization of scientific knowledge has always been the most important way of popularizing science. For example, the China Association for Science and Technology holds the National Science Popularization Day every year. It can be said that the National Science Popularization Day is the science popularization activity with the largest number of participants and the widest coverage in China. In addition, many local science and technology associations under the charge of the China Association for Science and Technology have established educational work committees. These educational work committees jointly organize popular science and cultural activities with schools, technology companies, and museums. For example, museums and science and technology museums carry out visit experience, science lectures and exhibitions, and science and technology hand-made participation and interaction, so as to achieve the purpose of publicizing science knowledge, promoting patriotic culture, and stimulating patriotic emotions.

\subsection{Scientific and Technological Achievements Patriotism Education Base}

The construction of a patriotism education base is an important carrier for disseminating science knowledge, driving scientific and technological forces, and stimulating the interests of young people. It is a basic project to improve the overall quality of the whole nation and is of great significance to young people's establishment of lofty ideals and formation of values. For example, the Beijing Natural History Museum, the Chinese Aviation Museum, the Navy Shanghai Expo and other patriotic education bases hold a variety of practical activities, which can allow young people to clearly and 
comprehensively recognize the high-tech achievements and technologies that China has achieved.

\subsection{Short Video on Popularization of Scientific Knowledge}

In the context of the converged media era, short videos have quickly become the main way for people to obtain various information and resources. The speed of video dissemination is fast, the scope is wide, and the dissemination effect is immediate. Therefore, various units, bases, and some selfmedia have seized the mainstream of new media and opened video accounts one after another to conduct online scientific knowledge popularization activities. For example, the China Association for Science and Technology opened a popular science China account on the Tik Tok platform. Compared with traditional offline activities, online science popularization activities break the limitations of time and space, attract more people of different ages to participate in the event, and can promote the latest popular science knowledge, achieving a very good publicity effect.

\subsection{Problems in the Current Publicity Path}

Popular science and cultural activities and scientific and technological achievements education bases are both offline activities. Although offline activities are more clear and intuitive, they are limited by time and space, and the number of activities is limited; In areas with backward economy and infrastructure, the number of popular science activities will also decrease; Participants are mainly experts in the field of science and technology, technology companies, governments, schools and other organizations, and the participation rate of ordinary people is low; Moreover, such activities and lectures are highly professional and scientific, and some event personnel may not understand it, making it difficult to accept.

Online science popularization activities such as short videos have solved the problems that occurred in offline activities, but the advantages of offline science popularization activities cannot be shown in online activities: For example, the form of popular science is singular and lack of innovation; short videos are too short and the content is not clear; Some selfmedia have insufficient knowledge and level, and mistakes in popular science, etc., which will seriously affect the effect of popular science activities.

\section{Design Strategy of Cultural and Creative Products Based on Scientific and Technological Achievements}

In view of the insufficiency of conducting online and offline popular science publicity activities, designing scientific and technological achievements and cultural and creative products to open up a new path for publicity activities: Interspersing scientific and technological cultural and creative products into online and offline popular science publicity activities can increase the participation of ordinary people and mobilize the enthusiasm of event personnel. Excellent cultural and creative products are not only novel and beautiful, but also can enter all aspects of people's lives and win the love of more ordinary people.

\subsection{Oriented by Public Demand}

Science and technology are not illusory, and the achievements of science and technology exist in the lives of ordinary people. Therefore, these cultural and creative products should not just be ornaments, but objects that people use every day and are used more often. For example, the T-shirts that people wear, cups for drinking water, canvas bags, mobile phone cases, etc., are the most common and easiest cultural and creative products to make. These objects are frequently used and updated frequently, which will prompt these cultural and creative products to be updated frequently to meet the needs of the public. In this way, the public will be exposed to the popularization of scientific and technological achievements in the process of use, and form a subtle understanding of scientific and technological achievements.

\subsection{Pay Attention to the Development of Materials for Scientific and Technological Achievements}

When designing cultural and creative products, there are various types of scientific and technological achievements, and each type of achievement is diverse. How to choose the appropriate result is a key issue in cultural and creative design: Choose the scientific and technological achievements that are often broadcast in the news, and these achievements are closely related to people's lives, and then flatten the design, give it bright and concise colors, and use large characters to explain; The specific results should be written on the cultural and creative products in short, so that when people use them, they can see the scientific and technological achievements made by our country at any time.

At present, my country's most important scientific and technological achievements are in the fields of manned spaceflight, deep-sea exploration, and navigation systems. The average age of key positions on the "Chang'e 5" team is only 33 years old, and the average age of the "Fighter" team is only 34 years old. The young generation of China has ideals and responsibilities, the country has a future, and the nation has hope, and there is a steady stream of powerful forces to achieve the goal of development. These teams are role models for young people, inspiring young people to assume historical responsibilities and contribute to the great rejuvenation of the Chinese nation. Therefore, this article selects three achievements in scientific research: "Chang'e-5", "Fighter" and "BeiDou Navigation Satellite System". These three achievements were made into t-shirts, named Wear the Chinese Dream on One's Body, to let more young people know the story of scientific research, understand the great achievements of our country and the enlightenment that these achievements have brought to young people. Cultural and creative products also include canvas bags and mobile phone cases, named Putting the Chinese Dream in Your Hands. These simple cultural and creative products can be quickly spread among young people. If the patterns are beautiful, fashionable and attractive, young people are very happy to wear them and hold them in their hands. 


\section{Design Practice of Scientific and Technological Achievements, Cultural and Creative Products}

\section{1 "Fighter" Pattern Creation}

On October 27, 2020, the "Fighter" successfully dived in the Mariana Trench to break through 10,000 meters to reach 10,058 meters, setting a new record for China's manned deep diving. At 8:12 on November 10, the "Fighter" successfully sat on the bottom of the Mariana Trench with a bottom depth of 10,909 meters, setting a new record for China's manned deep diving. On November 28, the President of China sent a letter to congratulate the "Fighter" all-sea deep manned submersible for successfully completing the 10,000-meter sea trial and returning to the voyage successfully.
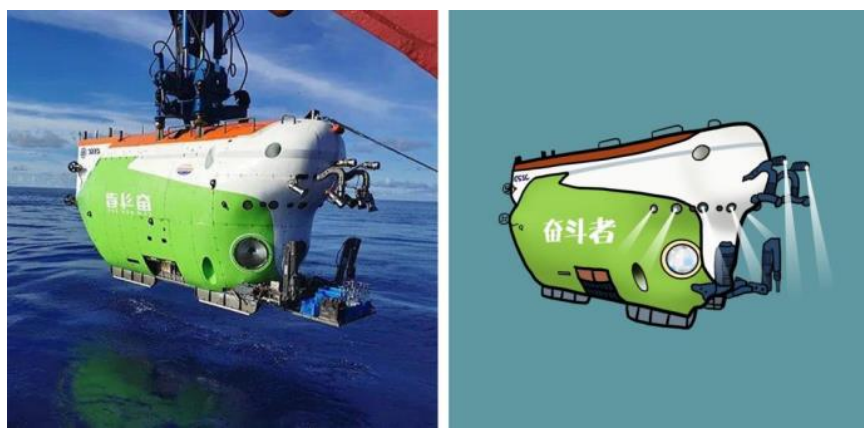

Figure 1: Prototype and design pattern of "Fighter"

The background of the "Fighter" pattern adopts the gray blue of the sea, the color is shallow but the brightness is low, showing the vastness of the depths of the sea; The fuselage is bordered by thick black lines, using the colors of the original equipment, with bright green and white as the main colors, which is full of vigor and power; When working in the deep sea, the headlights of the "Fighter" will be turned on, so the lights of the fuselage are turned on in the pattern to create a working atmosphere; As a scientific research result that is still in the confidential stage, the "Fighter" fuselage has many round holes. Although I don't know what these round holes represent, you can use your imagination to use the largest round hole of the fuselage as the window of the device. It is set to transparent white. Later, in the process of improvement, the white windows were transformed into light blue with bubbles. At this time, the windows looked more like eyes, adding vitality to the whole picture. At the same time, the square hole under the fuselage was transformed into a doorway to enter the equipment. In this way, the whole picture has unity and integrity, a house like a submersible, arousing people's thoughts and imagination.

\subsubsection{Cultural and creative T-shirt design}

Print the finished pattern on the T-shirt. Cultural and creative T-shirts are both common and simple cultural and creative products. At the same time, T-shirts have greater advantages: Everyone wears a T-shirt, and the cultural and creative patterns are printed on the T-shirt, which can play a good role in publicity. Compared with other cultural and creative products, the T-shirt has a better publicity effect. The term "Fighter" has a stimulating effect on raising young people's awareness of scientific research results and planning for their own future.

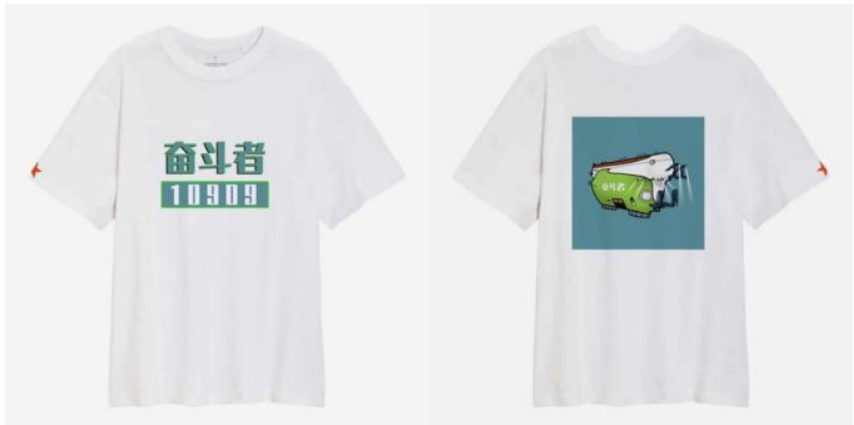

Figure 2: "Fighter" T-shirt design

This T-shirt has text on the front and a pattern on the back. The font of "Fighter" adopts lake green, and then uses bright green for bordering, and after adding shadows, the text effect is finally formed; Write "10909" below the font of "Fighter". This is the depth of the dive of the submersible and represents its achievements; There is a five-pointed star where there is a shoulder. The five-pointed star symbolizes organization and red power. On the back, the "Fighter" logo is set on the upper part of the middle of the T-shirt. The entire T-shirt is neat and generous, in line with the aesthetics of young people, and the term "Fighter" can resonate with young people: In the context of the new era, generations of young people continue to struggle to gain the strength of the country. The term "Fighter" is the label of all young people. The young people are practicing the title of "Fighter" with practical actions. Therefore, this T-shirts meet the psychological needs of young people.

\subsubsection{Design of cultural and creative mobile phone case}

Remove the background of the pattern and print it on the phone case to complete the production of the phone case. Simply draw a few wavy lines on the top of the pattern to represent the waves, indicating that the "Fighter" has dived into the deep sea and is working; Write the word "Fighter" under the pattern and write its diving depth; the font design is too small, leaving enough display space for the pattern.

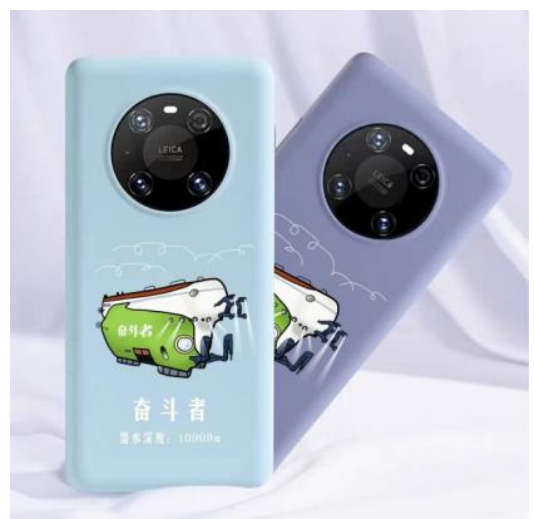

Figure 3: Mobile phone case design

\subsubsection{Cultural and creative canvas bag design}

Canvas bags are very common cultural and creative products. Similarly, the promotional effect of canvas bags is also very obvious. The design is reduced and printed in the center of the white canvas bag, and the canvas bag is left blank in a large area, which is very simple; The back of the canvas bag continues to be decorated with text, using the "Fighter 10909" style, the text is set in italics, and underlined to indicate 
emphasis.

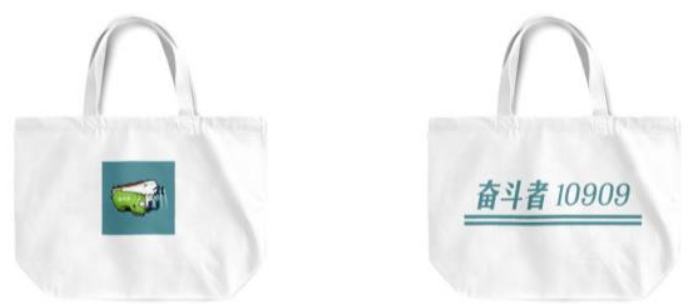

Figure 4: Canvas bag design

\section{2 "Chang'e 5" Pattern Creation}

At 4:30 on November 24, 2020, the Chang'e 5 carrier rocket was successfully launched at the Wenchang Space Launch Site in China. 23 days later, in the early morning of December 17 , after passing through many difficulties including lunar landing, automatic sampling, lunar take-off, lunar orbital rendezvous and docking, and reentry and return, the tested Chang'e-5 returner carried lunar samples (About $2 \mathrm{~kg}$ ), successfully landed in Siziwang Banner, Inner Mongolia.
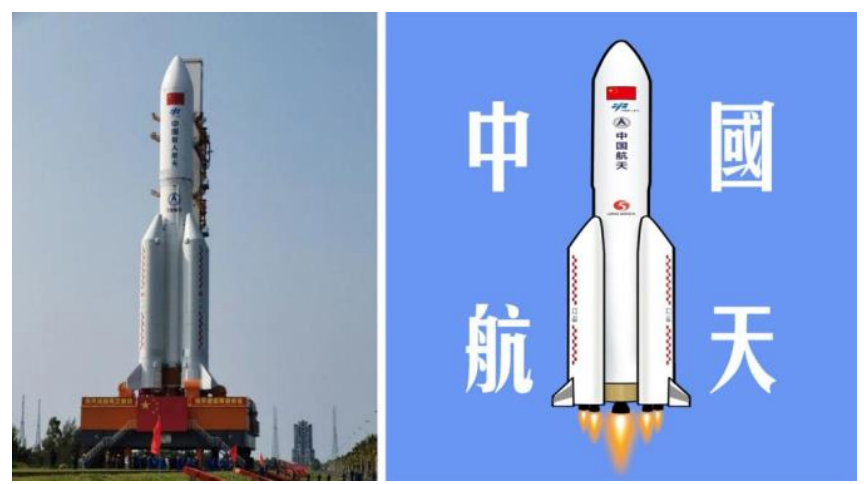

Figure 5: China Aerospace Pattern Design

The background of China's aerospace pattern selects sky blue to represent the vast sky, and flames are spraying from the bottom of the rocket, which symbolizes the Long March V traveling in infinite space; in reality, the entire rocket is a slender and long shape. In the process of design, the rocket is stretched left and right to deform it to become a short and fat cute image, which increases its sense of joy; The rocket is bordered by a black line, and the white rocket stands out against the blue background; Finally, the four words "China Aerospace" are written around the rocket. These four characters are evenly distributed around the rocket. The font size is too large to highlight the power of China's aerospace.

\subsubsection{Cultural and creative T-shirt design}

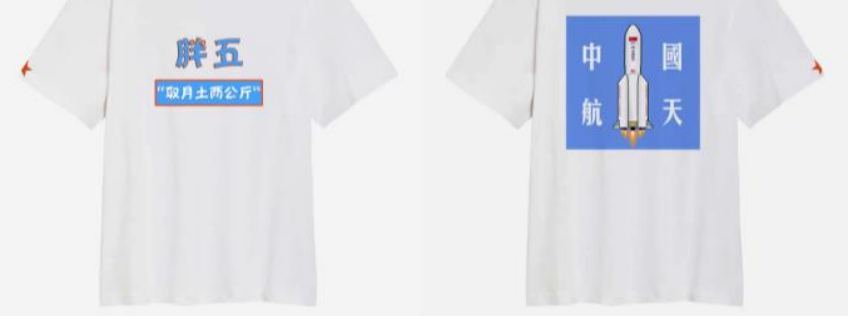

Figure 6: China aerospace T-shirt design

This T-shirt takes the form of text on the front and a pattern on the back. The "Fat Five" font adopts a rounded font, and the font color is sky blue as the background color. Since the Long March No. 5 rocket is printed with a five-star red flag, the red font of the red flag is bordered by the blue font to form a color contrast; The back of the T-shirt is the same as the previous design, with the map attached in the same position. The entire T-shirt is clean and tidy, which is very attractive to young people.

\subsubsection{Design of cultural and creative mobile phone case}

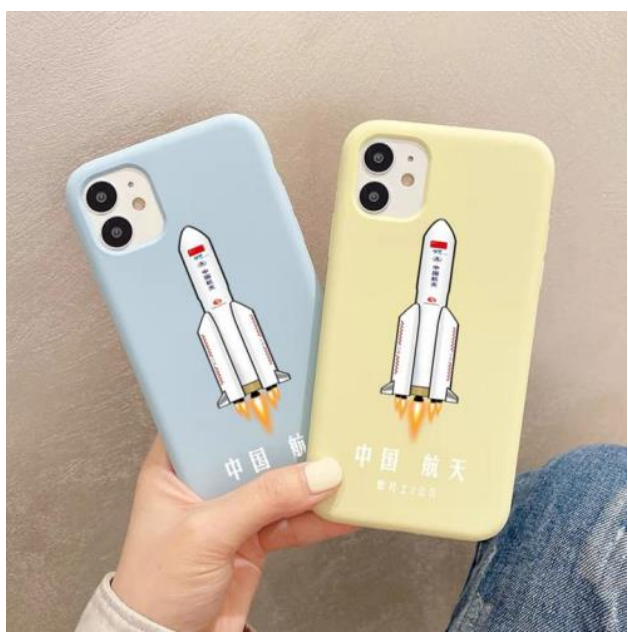

Figure 7: Mobile phone case design

Remove the sky blue background and fonts in the pattern, and only stick the rocket graphic on the phone case. Set "China Aerospace" under the rocket graphics, and there should be a space between "China" and "Space"; write "Take two kilograms of moon earth" under "China Aerospace", and the font setting is too small.

\subsection{BeiDou Navigation Satellite System Pattern Creation}

On June 23, 2020, Beidou-3's last global networking satellite was ignited and launched at the Xichang Satellite Launch Center. So far, the deployment of the Beidou-3 global satellite navigation system constellation has been fully completed. At 10:30 on July 31, 2020, the President of China announced the official opening of the BeiDou Navigation Satellite System.

The Beidou navigation system has a total of 55 satellites orbiting the earth. Therefore, the pattern created is centered on the earth, and many satellites move around the earth along the orbit. Among them, the background of the picture adopts the pop color system, the color block in the upper left corner (previously red) is changed to the vast starry sky, the other three color blocks are sky blue, pink and cobalt blue pop colors, four color blocks Evenly share background: The cartoon version of the Earth is located in the center of the picture, showing the Eurasian continent, Atlantic Ocean, Indian Ocean and other plates. The color of the plates also adopts the color of POP art, the Asian plate is green, the African plate is yellow, and the outline of the earth is bordered by thick black lines; Six white orbital lines revolve around the earth in different shapes. Each orbital line has 1-2 satellites orbiting the earth, and the shapes of the satellites are also different; The whole pattern is brightly colored, clean and beautiful. 


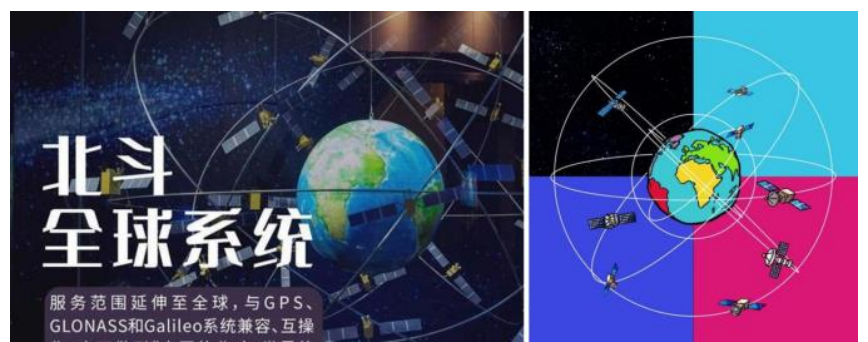

Figure 8: "Beidou Navigation" pattern design

4.3.1 Cultural and creative T-shirt design

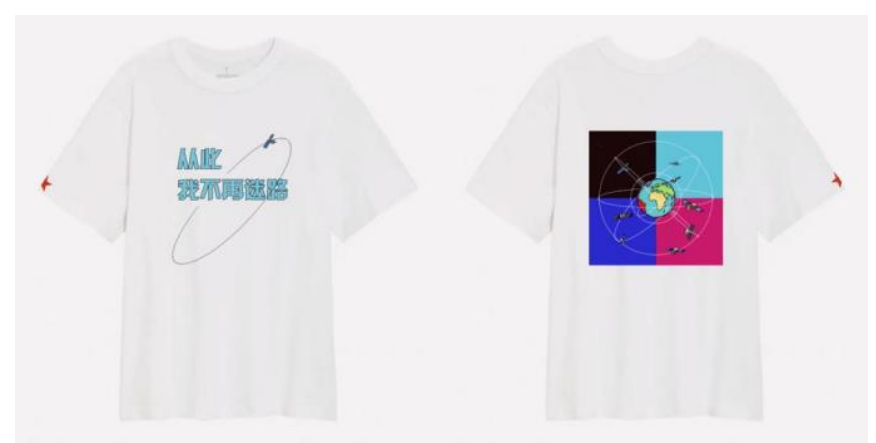

Figure 9: "Beidou Navigation" T-shirt design

Similarly, this T-shirt takes the form of a front text and a back graphic. For the public, the biggest purpose of "Beidou Navigation" is to accurately locate, so that everyone is no longer lost. Therefore, the front text is divided into two lines with the words "I'm no longer lost", "I" is a single line, "No more lost" is the second line, the color is light sky blue, and the black thin line is used as the text Carry out edge processing; A black circle is hung on the upper right corner of the word "road". A satellite is moving on the circle, indicating that the reason why people are no longer lost is because of the Beidou navigation system.

\subsubsection{Design of cultural and creative mobile phone case}

After removing the pattern background, stick it on the phone case. Write the words "Beidou Navigation" below the pattern, and there is a space between "Beidou" and "Navigation"; Print "ZHONG GUO" below the text, and write the year: 2021 in the center of the bottom. The whole phone case is clean, bright, and the theme is clear, suitable for young people.

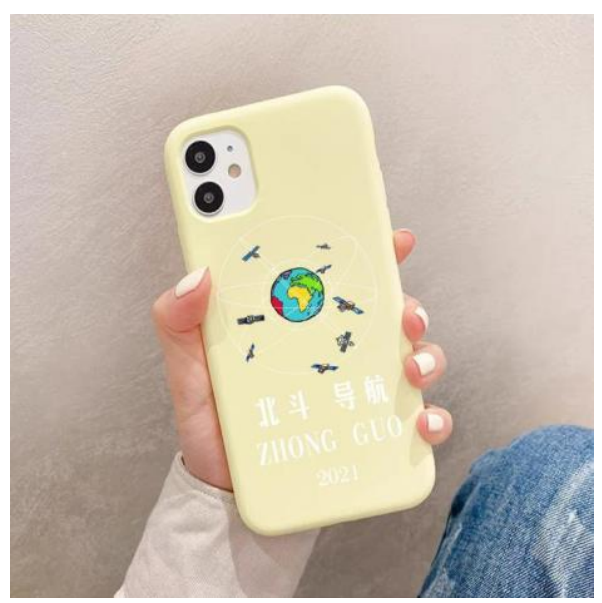

Figure 10: Mobile phone case design

\section{Conclusion}

This article is based on the social issue of how to make ordinary people better understand the major scientific and technological achievements that China has made at present. Analyzed the current scientific and technological achievements publicity path and its shortcomings, and proposed to popularize scientific knowledge and publicize scientific and technological achievements by designing scientific and technological achievements and cultural and creative products, which not only effectively improves the publicity effect, but also provides ideas for the design of other cultural and creative products. Cultural and creative products based on these important scientific research results can better attract young people to increase their patriotic awareness, understand major events in the country, and enhance national self-confidence. Therefore, it is necessary to create a batch of cultural and creative products that can express these scientific research achievements made by countries in the new era, and to open up new paths for enhancing cultural confidence and telling Chinese stories well.

\section{References}

[1] Fei Xi, Xuejun Ruan. Research on the design of cultural and creative products based on aerospace culture[J]. Art Education Research, 2021(05): 70-74.

[2] Yu Ma, Xuerui Wang. The design and application of terracotta warriors and horses in cultural and creative products[J]. Packaging Engineering, 2020, 41(14): 304310.

[3] Ge Gao. Research on Cultural Creative Product Design Based on Product Gene[D]. Harbin Engineering University, 2019.

[4] Qing Lu. Research on the Design and Application of Cultural Creative Products in Colleges and Universities Based on Design Psychology[D]. Zhengzhou University, 2019. 